\title{
Femtosecond laser-induced melting and shaping of indium nanostructures on silicon wafers
}

A. Azarm, F. Akhoundi, R. A. Norwood, N. Peyghambarian, and K. Kieu

Citation: Appl. Phys. Lett. 113, 033103 (2018); doi: 10.1063/1.5026707

View online: https://doi.org/10.1063/1.5026707

View Table of Contents: http://aip.scitation.org/toc/apl/113/3

Published by the American Institute of Physics

\section{Articles you may be interested in}

Transparent $\mathrm{ZnO}: \mathrm{Al}_{2} \mathrm{O}_{3}$ films with high breakdown voltage and resistivity

Applied Physics Letters 113, 032102 (2018); 10.1063/1.5028513

Patterning of graphene oxide with optoelectronic tweezers

Applied Physics Letters 113, 031106 (2018); 10.1063/1.5025225

Stress mapping of a strain superlattice using scanning moiré fringe imaging

Applied Physics Letters 113, 031905 (2018); 10.1063/1.5022842

Direct imaging of surface states hidden in the third layer of $\mathrm{Si}(111)-7 \times 7$ surface by $\mathrm{p}_{\mathrm{z}}$-wave tip

Applied Physics Letters 113, 031604 (2018); 10.1063/1.5038954

Bubble dimer dynamics induced by dual laser beam ablation in liquid

Applied Physics Letters 113, 021902 (2018); 10.1063/1.5030437

Exclusion of injection efficiency as the primary cause of efficiency droop in semipolar ( $20 \overline{2} \overline{1})$ InGaN/GaN lightemitting diodes

Applied Physics Letters 113, 031101 (2018); 10.1063/1.5036761

\section{LakeShore CRYOTRONICS}

\section{Sensors, Controllers, Monitors}

from the world leader in cryogenic thermometry 


\title{
Femtosecond laser-induced melting and shaping of indium nanostructures on silicon wafers
}

\author{
A. Azarm, ${ }^{\text {a) }}$ F. Akhoundi, R. A. Norwood, N. Peyghambarian, and K. Kieu \\ College of Optical Sciences, The University of Arizona, Tucson, Arizona 85721, USA
}

(Received 22 February 2018; accepted 3 July 2018; published online 17 July 2018)

\begin{abstract}
We study the modification of indium semi-spherical nanostructures with radii of around $175 \mathrm{~nm}$ on silicon wafers into linear microstructures more than $2 \mu \mathrm{m}$ long in the direction of polarization of laser pulses ( $1.56 \mu \mathrm{m}, 150 \mathrm{fs}$, up to $7.5 \mathrm{~nJ}$ and 30000 laser pulses with $8 \mathrm{MHz}$ repetition rate). The experimental results and a rudimentary analysis confirm that melting occurs from intense laser pulses. In short, we demonstrate that melting of the indium droplet followed by trapping in high spatial frequency laser induced periodic surface structures on a silicon substrate cause nanostructure modification. The understanding of the modification process, melting, and moving in the nano-grating structured field, pave the way to design nanostructures of arbitrary shapes at the sub-wavelength scale. Published by AIP Publishing. https://doi.org/10.1063/1.5026707
\end{abstract}

Nanostructures are being used to harness and manipulate light for many applications. Namely, periodic arrays of subwavelength plasmonic nanostructures, metasurfaces, provide advanced wavefront engineering and waveguiding that cannot be found in nature. ${ }^{1}$ Making such nanostructures is a challenge. One method to make an arbitrary array of nanostructures is based on control at the growth stage like the electron beam lithography technique. Another method is based on post growth control, with femtosecond (fs) laser material processing being one of the prevalent post growth techniques. ${ }^{2}$ fs lasers have been used widely to modify nanostructures, for instance, for nanojet or nanobump creation, ${ }^{3}$ modification of spherical silver nanoparticles embedded in glass to prolate and oblate spheroids, ${ }^{4}$ three dimensional self organization of metallic nanostructures, ${ }^{5}$ etc.

The phenomenon of laser induced periodic surface structure (LIPSS) formation is key for many fs laser material modification processes like three dimensional self organization in nanocomposite layered systems. ${ }^{5}$ In addition, LIPSS itself has been used for nano scale surface modification (see the recent review papers of LIPSS ${ }^{6,7}$ ). In the first observations of LIPSS, the ripple had a period almost the same as the wavelength of the incident laser pulses. ${ }^{8}$ This is known as the low spatial frequency LIPSS (LSFL). ${ }^{9}$ With the use of fs lasers, nano-ripples have been observed, which is known as high spatial frequency LIPSS (HSFL) (see Refs. 6 and 7). The period of nano-ripples, nano-grating, could be as small as $\Lambda=\lambda / 2 n^{*}$ in which $\lambda$ is the wavelength of the incident laser and $n^{*}$ is the modified refractive index of the surface material. ${ }^{10}$ The grooves direction of nano-grating depends on the polarization direction of laser pulses.

In this paper, we will show a method to perform subwavelength nanostructure modification by means of two effects (melting and HSFL) in fs laser pulse interaction with nanostructures. In particular, we demonstrate experimental results on a possible physical mechanism underlying the observed nanostructure modification. We elaborate on the recently reported phenomenon of fs laser modification of

a)aazarm@email.arizona.edu nanostructures. ${ }^{11}$ In addition to the experimental results, a basic analysis of heating from absorption indicates that an indium droplet is melted either by single laser pulse irradiation or by heat accumulation due to multiple pulse irradiation depending on the pulse energy. It is confirmed experimentally that HSFL plays a major role in nanostructure shaping after melting, indicating that this method of melting and shaping nanostructures is a promising tool for making metasurfaces.

The nanostructures are grown with a condensed source molecular beam epitaxy system (MBE32 Ribber) ${ }^{11}$ on the two inch $\mathrm{n}$-doped(P)-Si(111) substrate from Siltronix. The growth procedure is based on a vapor-liquid-solid (VLS) technique which is commonly used to grow nanowires. ${ }^{12}$ Briefly, first an indium droplet is created. Then at higher temperature, a mixture of the indium droplet and the gas phase of Ga and As creates an alloy. Precipitation of GaAs occurs while the concentration of GaAs in the created alloy increases to the saturation level. Therefore, the nanowire of GaAs grows at the bottom of the alloy.

The preparation of the substrate was done as in Ref. 11, with growth being started at an elevated temperature of around $250^{\circ} \mathrm{C}$. Sixteen monolayers (MLs) of indium, based on calibration of the beam equivalent pressure to planar deposition rate, were deposited on the $\operatorname{Si}(111)$ surface. Then, 16 MLs of an arsenic cap layer were deposited to avoid reevaporation of indium in the following stage. The cap layer was desorbed in the following stage by heat treatment up to $460{ }^{\circ} \mathrm{C}$. At the end of seven minutes of heat treatment, $\mathrm{Ga}$ and As cell's shutters were opened for a period of time corresponding to 16 MLs of GaAs deposition. The temperature of the substrate was measured with a thermocouple. One can grow GaAs nanowires by extending the last step of the GaAs flux time, and the use of the $\mathrm{Si}(111)$ substrate compared to the $\operatorname{Si}(100)$ substrate $^{11}$ resulted in an additional hexagonalshaped base of the intact nanostructure with a dome-shaped droplet on the top (see Fig. 1). The density of nanostructures was around 0.5 nano-particles per square micrometer and the radius of the semi-sphere was distributed around $175 \mathrm{~nm}$.

$\mathrm{An}^{3+}{ }^{3+}$-doped femtosecond fiber laser mode-locked by a single-walled carbon nanotube saturable absorber is used 


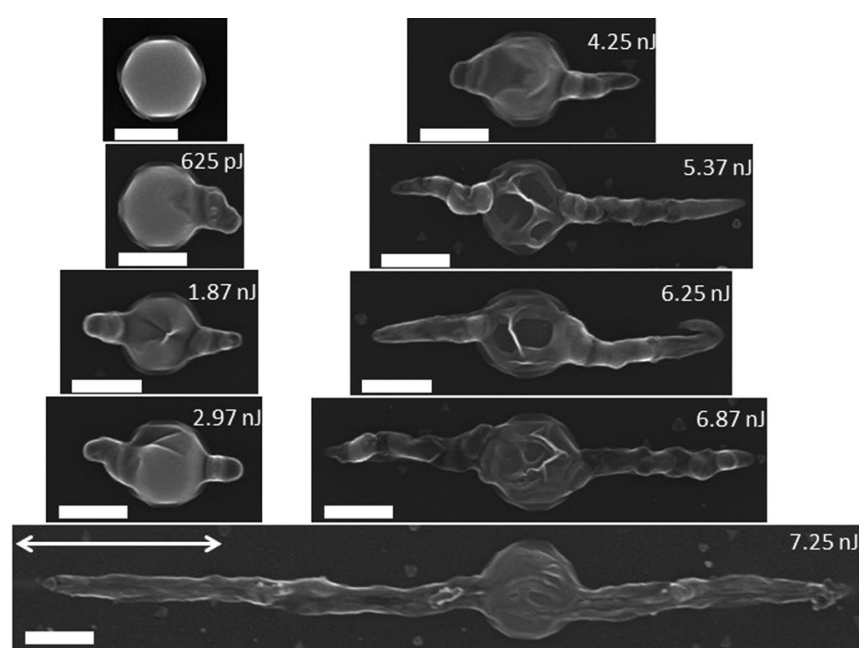

FIG. 1. Scanning electron microscope (SEM) images of intact (top left) and irradiated nanostructures with $\sim 13000$ laser pulses with linear polarization (from left to right as indicated by a double-sided arrow). The energy of laser pulses is shown in the upper right part of each image except for the intact nanostructure. The hexagonal base is clearly visible in the intact nanostructure on the $\operatorname{Si}(111)$ substrate. The scale bar is $250 \mathrm{~nm}$ in all SEM images.

to modify the grown nanostructures. The laser is part of a multiphoton microscope as introduced in Ref. 13. We verified that the laser beam was focused at the substrate surface by third harmonic generation (THG) imaging of the surface. The laser output is linearly polarized with a repetition rate of around $8 \mathrm{MHz}$ and a maximum pulse energy of $8.5 \mathrm{~nJ}$. The overlap of pulses at the focal point is controlled by a galvo mirror raster scan time. The focal point spot size is $1.86 \mu \mathrm{m}$, measured by the THG knife edge method. The pulse duration is around 150 fs at the full width at half maximum of the intensity profile measured by an autocorrelator. The peak intensity is estimated at around $0.83 \mathrm{TW} / \mathrm{cm}^{2}$ for pulses with an energy of $7.25 \mathrm{~nJ}$. The energy of the laser pulse is varied with a polarizer and a half waveplate. An FEI Helios Nanolab 660 DualBeam electron microscope focused ion beam (FIB) was used to collect the image of intact and modified nanostructures.

Figure 1 shows the scanning electron microscope (SEM) images of original and irradiated hexagonal-shaped nanostructures on $\mathrm{Si}(111)$. The energy of the linearly polarized fs laser pulses is shown in the upper right part of each image. Each individual nanostructure was exposed to $\sim 13000$ pulses. The indium droplets were ablated for laser pulses with energies above $7.5 \mathrm{~nJ}$ (not shown).

Figure 2 (square) shows the elongation obtained by examining each single extended nanostructure versus laser pulse energy. The elongation is calculated as the nanostructure's diameter subtracted from the extended length. Figure 2 (circles) demonstrates the extension of nanostructures versus the number of pulses for fixed energy $(2.5 \mathrm{~nJ})$. We did a similar experiment with higher fixed energy $(6.25 \mathrm{~nJ})$ which is not shown here. The trend is similar as Fig. 2 (circles). We observe that elongation varies linearly with the number of pulses for a very low number of pulses (less than 3000) as shown in Fig. 2 (circle). We can predict the effect of each pulse from the slope of the linear fit to the curve in the low pulse number regime of Fig. 2 (circle). The slope is found to

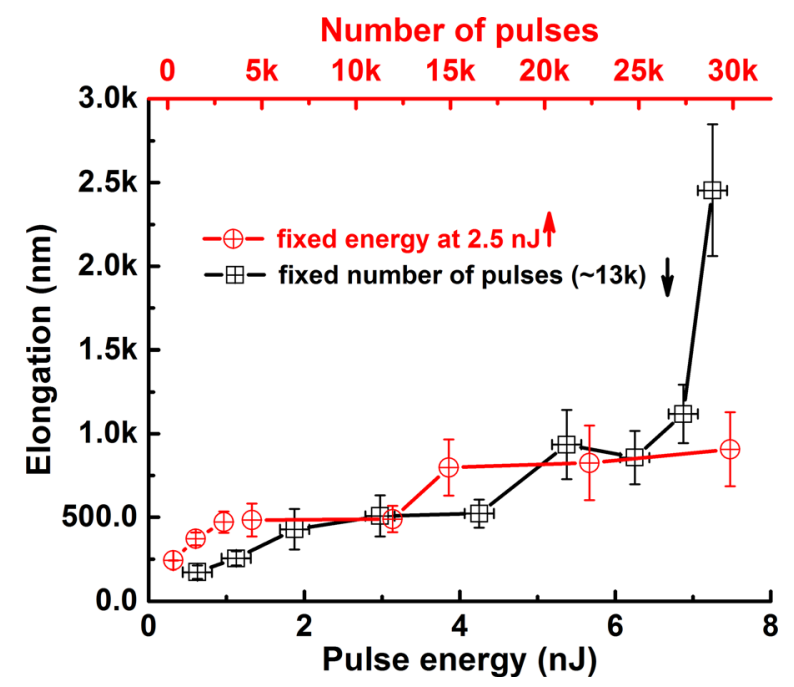

FIG. 2. Average elongation over more than hundred exposed nanostructures is plotted versus energy of incoming laser pulses at a fixed number of pulses (square). The average length of many modified nanostructures versus the number of pulses for a fixed laser pulse energy of $2.5 \mathrm{~nJ}$ (circle). The arrows in the legend indicate the designated $\mathrm{x}$ axis.

be around $100 \mathrm{pm}$ per pulse. The slope increases to around $350 \mathrm{pm}$ per pulse for a pulse energy of $6.25 \mathrm{~nJ}$.

Still, there is subtle information in the SEM images of Fig. 1. The elongation occurs in a small part of the nanostructure; further, the thickness of the modified part is distributed around a fixed value $(\sim 100 \mathrm{~nm})$ which is independent of the incoming pulse energy or the number of pulses. This implies that there is a physical mechanism forcing the material to be extended in a small preferred part of the nanostructure in the laser pulse's polarization direction. In the next paragraphs, we will discuss how HSFL defines the thickness of the extended materials or the nano-grating period on the surface. One may imagine that extension by the laser pulse has a preferred direction because the nanostructure has a crystalline shape and there is a preferred growth orientation. We rotated the substrate relative to the laser polarization direction in the multiphoton microscope. The results show that there is no prefered direction other than the laser's polarization direction, this is an indication that the elongation direction only depends on the polarization direction of the incoming pulses.

In order to understand the role of the substrate surface on the modification process, nanowires of GaAs with an average height of $500 \mathrm{~nm}$ are grown by extending the last growth step in our VLS technique. The grown nanostructures are similar to the intact nanostructures shown in Fig. 1 except that the base height is around $500 \mathrm{~nm}$ above the substrate surface. There are also some nanowires which have grown horizontally on the surface of the substrate [Fig. 3(a)]. The height of the nanowires is much smaller than the Rayleigh range ( $\sim 7 \mu \mathrm{m})$ of the focused laser beam. The nanowire sample is illuminated by focused fs laser pulses having linear polarization with different energies and number of pulses. Only the nanowires that were grown in the plane of the substrate surface were extended in the laser polarization direction [Fig. 3(b)]. However, the droplets of those nanowires protruded from the surface vertically, remained intact, or dropped off around the base of the nanowires. The observation implies 

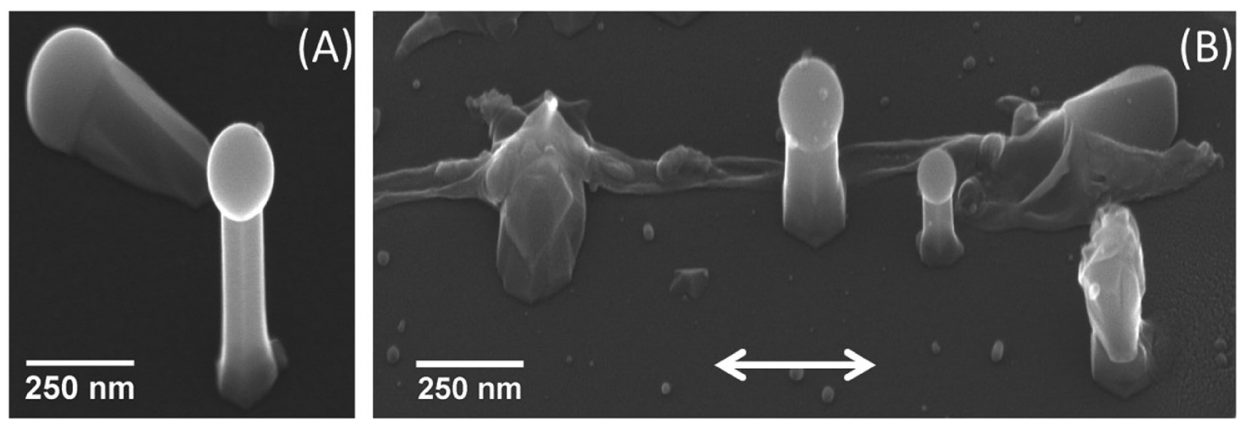

FIG. 3. (a) SEM image of the intact GaAs nanowire with indium droplet on the top (grow vertically and on the surface). (b) Exposed nanowires with 3 nJ energy and $\sim 1500$ laser pulses with linear polarization (from left to right as indicated by a double-sided arrow). The indium droplets on the GaAs nanowire in the center and the smaller one on the right side of it stayed intact and the one on the lower right dropped onto the GaAs base as it was melted. The other two indium droplets on the surface were modified as shown in Fig. 1. Both SEM images were obtained at a tilt angle of $45^{\circ}$.

two important conclusions. The first one is that the droplets were melted by laser pulses, and the second is that the substrate surface must play a role. Note that the crystalline GaAs base was not modified and that the only modified part is the indium droplet attached to the GaAs base.

Indeed, a rudimentary analysis indicates that the indium droplet will be melted at a particular energy of a single laser pulse while other materials like GaAs or Si remain intact. First, because GaAs and Si have a very small absorption coefficient at the laser wavelength $(1.56 \mu \mathrm{m}) .{ }^{14}$ Note that the absorption of $\mathrm{GaAs}$ or $\mathrm{Si}$ at the second harmonic generation (SHG) or THG wavelength is high, whereas the conversion efficiency is very low. Therefore, the total absorption by $\mathrm{Si}$ or GaAs is negligible. Second, the melting temperatures of $\mathrm{GaAs}$ and $\mathrm{Si}$ are very high $\left(1238\right.$ and $1414^{\circ} \mathrm{C}$, respectively $)^{15}$ unlike indium $\left(156.59^{\circ} \mathrm{C}\right) .{ }^{15}$ Note that the indium droplet was mixed with other metals like Ga or As to make an eutectic alloy in the growth process. Consequently, the In and Ga alloy may have a lower melting point than indium. The following calculation shows that the melting of the indium droplet is plausible. A laser pulse with $5 \mathrm{~nJ}$ energy and uniform power distribution on a circle with a radius of $1.86 \mu \mathrm{m}$ delivers $44 \mathrm{pJ}$ of energy to a nanostructure with $175 \mathrm{~nm}$ radius. The assumption of uniform power distribution is not accurate because the laser beam has a Gaussianlike distribution. As a result, the assumption underestimates the final absorbed energy by a nanostructure. Regarding the reflection of indium (93\% at normal incident) at near infrared $(1.56 \mu \mathrm{m})$, the rest of incident radiation $(7 \%$ of $44 \mathrm{pJ} \sim 3.0$ $\mathrm{pJ})$ is absorbed by a thin layer of indium defined by the penetration depth $(\sim 10 \mathrm{~nm})$. The absorption coefficient of indium is $9.24 \times 10^{5} \mathrm{~cm}^{-1}$ at $1.56 \mu \mathrm{m} .{ }^{14}$ Note that $99 \%$ of transmitted energy $(3.0 \mathrm{pJ})$ is absorbed in a layer near the surface as thick as $50 \mathrm{~nm}$. The mass (m) of $50 \mathrm{~nm}$ thick indium on a semi-sphere nanostructure with a radius of $175 \mathrm{~nm}$ is $52 \mathrm{fg}$ (the indium density is $7.31 \mathrm{~g} / \mathrm{cm}^{3}$ ). ${ }^{15}$ Indium's specific heat capacity $\left(c_{p}\right)$ and latent heat of fusion $\left(l_{f}\right)$ are $0.23 \mathrm{~J} / \mathrm{gK}$ and $28.45 \mathrm{~J} / \mathrm{g}$, respectively. ${ }^{15}$ Therefore, the total required energy (Q) to melt $52 \mathrm{fg}$ of indium droplet from room temperature $\left[Q=m c_{p}(131.6 \mathrm{~K})+m l_{f}\right]$ is $3 \mathrm{pJ}$. Hence, the indium nanostructure is melted by a single fs pulse of $5 \mathrm{~nJ}$ energy assuming no thermal loss. For lower pulse energy, heat accumulation may cause the melting process to occur. The calculation of exact heat accumulation and transfer is beyond the scope of the current paper. We assume that the nanostructure consists of only $\mathrm{Si}$ and absorbs like indium, because silicon has the largest thermal conductivity $(1.24 \mathrm{~W} / \mathrm{cm} \mathrm{K})^{15}$ and diffusivity $\left(0.853 \mathrm{~cm}^{2} / \mathrm{s}\right)^{15}$ amongst nanostructure materials. This assumption underestimates the amount of heat accumulation. We calculated the heat accumulation using the analytical expression given in Ref. 10 for the lowest energy of 625 pJ. Only 2500 pulses are required to reach an equivalent temperature to melt indium. Hence, a repetitive laser pulses with lower energy $(<5 \mathrm{~nJ})$ melts the indium at a specific number of pulses. The approximate calculation and experimental evidences confirm that the fs laser melts the metallic droplet. Therefore, first fs laser pulses melt the indium droplet, then in the following step, the indium droplet is transformed to the modified structure.

We would like to verify whether the crystalline base, despite being left intact by the laser pulses, has an effect on the modification process. Hence, nanostructures without the last step in our VLS technique were grown, the same heat treatment was used as the GaAs was deposited. fs laser induced modification was also clearly observed, showing that the crystalline base does not play a role in the modification process.

In addition, we observed that the number of protruded extensions depends on the diameter of the nanostructure. For example, only one line emerges from the smallest nanostructure shown in Fig. 1, while two parallel lines came out of a larger nanostructure. Figure 4 shows three lines growing from a larger nanostructure after irradiation with 1500 linearly polarized laser pulses with an energy of $5.75 \mathrm{~nJ}$. The distance between extended lines is $249 \pm 17 \mathrm{~nm}$ by doing 2DFFT analysis over a few modified nanostructures. The thickness of each line is similar to the single extension cases $(\sim 100 \mathrm{~nm})$. Although this is very similar to LIPSS, it is not evident whether it is a LIPSS of the indium nanostructure or the silicon substrate. Therefore, we made a $350 \mathrm{~nm}$ thick indium thin film on the $\mathrm{Si}(111)$ substrate by e-beam evaporation. The ripples could be observed on the surface after irradiation with $\sim 60000$ linearly polarized laser pulses of $8 \mathrm{~nJ}$ energy. The ripples spacing is $1.15 \mu \mathrm{m}$ by 2DFFT analyses of SEM images. This is LSFL and very different from what has been observed with our nanostructures.

The other candidate for LIPSS is the silicon substrate itself. The refractive index of silicon at $1.56 \mu \mathrm{m}$ wavelength is $\mathrm{n}=3.47 .^{14}$ The effect of dopant concentration on the 


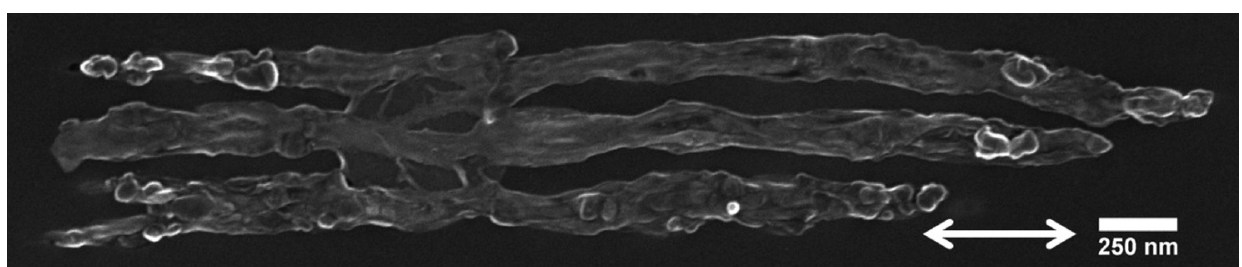

FIG. 4. SEM image of the large indium nanostructure (intact semi-sphere with $\mathrm{r} \sim 280 \mathrm{~nm}$ ) irradiated with (from left to right) linearly polarized (as indicated by a double-sided arrow) fs pulses, three rows of modified indium have a period of $249 \mathrm{~nm}$.

refractive index is minute. The modified refractive index $\left(n^{*}\right)$ includes the correction terms from the Drude $\left(n_{D}\right)$ and Kerr $\left(n_{K}\right)$ effects $\left(n^{*}=\sqrt{n^{2}+n_{K}^{2}+n_{D}^{2}}\right)$. At an intensity (I) of $0.66 \mathrm{TW} / \mathrm{cm}^{2}$ and silicon's nonlinear refractive index $\left(n_{2}\right)$ of $4.5 \times 10^{-14} \mathrm{~cm}^{2} / \mathrm{W},{ }^{16}$ the Kerr term $\left(n_{K}^{2}=2 n n_{2} I\right)$ is 0.2 . The modified refractive index increases by the inclusion of the Kerr effect, unlike the Drude effect. ${ }^{18}$

The Drude term reads $n_{D}^{2}=-\frac{e^{2} N_{e}}{m^{*} m_{e} \epsilon_{0}} \frac{\tau}{\omega} \frac{1}{\omega \tau+i}$ in which e and $m_{e}$ are electron charge and mass, respectively, $\epsilon_{0}$ is the vacuum permittivity, $\omega$ is the angular frequency of the incident wave, $m^{*}$ is the effective optical mass of the carrier, $N_{e}$ is the conduction band electron density, and $\tau$ is the Drude damping time. The silicon characteristics for the Drude correction term calculation are obtained from Ref. 10. The electron density used in the Drude model from single photon absorption is negligible due to the very low linear absorption coefficient of silicon. Although the two photon absorption coefficient of silicon is large $(\beta=0.5 \mathrm{~cm} / \mathrm{GW}),{ }^{17}$ we used the Keldysh formulation ${ }^{18}$ to find the carrier density in the conduction band because the Keldysh parameter is one at an intensity of $0.66 \mathrm{TW} / \mathrm{cm}^{2}$. In the presence of a strong external electric field, the Keldysh parameter is proportional to the ratio of tunneling time, from the valence to conduction band, to the period of the external electric field. Furthermore, the avalanche rate $\left(\gamma N_{e}\right)$ is considered plus the Keldysh rate $\left(W_{K}\right)$. In an avalanche process, the freed electrons gain more energy from the external field through the inverse Bremsstrahlung process and impact-excite more electrons into the conduction band. On the other hand, the critical plasma density defines the maximum attainable electron density. ${ }^{19}$ The plasma critical density is $\left(N_{c r}=\frac{m_{e} \epsilon_{0}}{e^{2}} \omega^{2}\right)$. At and beyond the plasma critical density $\left(\mathrm{N}_{c r}=4.5 \times 10^{20} \mathrm{~cm}^{-3}\right.$ at an incident laser wavelength of $1.56 \mu \mathrm{m})$, further absorption is inhibited. Therefore, the electron density is obtained by solving the following rate equation $\frac{\partial N_{e}}{\partial t}=\left(W_{K}+\gamma N_{e}\right)$ $\left(1-\frac{N_{e}}{N_{c r}}\right)$. The solution of the rate equation indicates that the minimum required pulse energy to achieve the critical electron density is $1.7 \mathrm{~nJ}$. Consequently, the Drude correction term $\left(n_{D}^{2}\right)$ is dominant and more importantly is fixed for a range of energy above $1.7 \mathrm{~nJ}$. The corrected HSFL period for the data in Fig. 4 is $\left(\lambda / 2 \mathrm{n}^{*}\right) \sim 256 \mathrm{~nm}$ and its very good agreement with the measured value implies that the modification process has the same origin as HSFL from the silicon substrate. The effect of multiple pulses or feedback effect in formation of HSFL needs to be considered to have a better agreement. As it is shown in Ref. 21, the nano-grating period and its tilt angle depends on the angle between the laser polarization direction and raster scan direction. In our work, the angle between the laser polarization direction and raster scan direction is zero. Therefore, the tilt angle is zero. In addition, the effect of nonhomogeneous heat diffusion on the width and period of HFSL needs to be considered in future work.

Although the numerical calculation is needed to describe wave scattering on the surface and HSFL formation as in Ref. 22 , a simple description give us qualitative analysis. In a simplified model, each semi-sphere is considered as a scatterer. This scatterer acts as a dipole and emits radiation on the Si surface. The scattering intensity is maximum at the direction perpendicular to the laser polarization direction. The interference pattern of the scattered field with incoming or scattering from another indium nanostructure is created. This defines the nano-ripples direction. Grating grooves are parallel to the polarization direction of laser pulses. In addition, the field enhancement occurs (stronger gradient) at the pole of the semi-spherical nanostructure which helps material movement at pole positions.

At the first glance, the modification process in our experiment is contrary to the explained mechanism of modification of spherical silver nanoparticles embedded in soda lime glass into the prolate spheroid. Briefly, the modification mechanism is described as follows: first, photoionization of the silver nanoparticle results in ejection of electrons on the poles of the nanosphere. The ionized silver atoms repel each other homogeneously around the nanosphere. The recombination of electrons and silver ions creates neutral silver atoms. The neutral silver atoms diffuse back to the nanoparticle and precipitate mainly at the poles. ${ }^{23}$ Currently, it is clear that the mechanism explained in Ref. 23 is operative in our case if we consider the nano-grating like scattered field pattern on the silicon substrate. It means the molten indium droplet moved in the polarization direction as explained in Ref. 23. Then the mold-like electric field structure (HSFL) traps the melted indium. As discussed in Ref. 20, the LIPSS of ns pulses can make an optical trap on the randomly distributed gold nanoparticle on silicon substrates and transform them into a well-defined periodic gold nanostructure. The gradient optical trapping force of HSFL increases using fs laser pulses. This nanostructure modification scenario is questionable regarding that the nanostructure of silver and gold has different thermos-physical and surface wetting properties than the indium droplet. An alternative scenario of nanostructure modification is as follows. It has been shown in Ref. 24 that the indium droplet created by the physical vapor deposition method tends to form in shallow surface depression created by fs laser ablation. In the case of nanostructure modification, field enhancement at the edge of the nanostructure in the nano-grating pattern results in local ablation of the substrate. The ablation of the substrate makes a shallow dip as in HSFL. Indium atoms obtain enough energy from laser pulses to move and consequently trap in the nano-groove structure. As we showed, melting is possible with the silicon surface ripple on the substrate playing a trapping role. Therefore, the melted material traps on the nano- 
grating frame created in the silicon and solidifies later. The HSFL is the central phenomenon of the modification process, defining the direction, thickness, and period of protruded extensions. Therefore, one can control the modification process by changing the polarization and wavelength of the incoming laser pulses.

We have presented the modification of the indium nanostructure by linearly polarized fs laser pulses at $1.56 \mu \mathrm{m}$ wavelength up to the ablation threshold. Moreover, the two important physical phenomena underlying the modification process are recognized as melting and HSFL. The modification process can be seen as follows: fs laser is used to first melt the nanoscale material and then shape the melted metals in the HSFL pattern.

The authors appreciate the support of the Air Force Office of Scientific Research (AFOSR, FA9550-15-1-0389) and fruitful suggestions of Dr. Gernot Pomrenke. A.A. thanks Professor Jerome V. Moloney, Dr. Colm Dineen, and Ms. Aoxue Han for the fruitful discussions. A.A. is thankful for the help of Dr. Paul Wallace at the imaging core facility of The University of Arizona.

${ }^{1}$ H.-T. Chen, A. J. Taylor, and N. Yu, "A review of metasurfaces: Physics and applications," Rep. Prog. Phys. 79, 076401 (2016).

${ }^{2}$ R. R. Gattass and E. Mazur, "Femtosecond laser micromachining in transparent materials," Nat. Photonics 2, 219 (2008).

${ }^{3}$ F. Korte, J. Koch, and B. N. Chichkov, "Formation of microbumps and nanojets on gold targets by femtosecond laser pulses," Appl. Phys. A 79, 879 (2004).

${ }^{4}$ A. Stalmashonak, G. Seifert, and H. Graener, "Optical three-dimensional shape analysis of metallic nanoparticles after laser-induced deformation," Opt. Lett. 32, 3215 (2007).

${ }^{5}$ Z. Liu, J. Siegel, M. Garcia-Lechuga, T. Epicier, Y. Lefkir, S. Reynaud, M. Bugnet, F. Vocanson, J. Solis, G. Vitrant, and N. Destouches, "Threedimensional self-organization in nanocomposite layered systems by ultrafast laser pulses," ACS Nano 11, 5031 (2017).

${ }^{6}$ R. Buividasa, M. Mikutisc, and S. Juodkazis, "Surface and bulk structuring of materials by ripples with long and short laser pulses: Recent advances," Prog. Quantum Electron. 38, 119 (2014).

${ }^{7}$ J. Bonse, S. Hohm, S. V. Kirner, A. Rosenfeld, and J. Kruger, "Laserinduced periodic surface structures a scientific evergreen," IEEE J. Sel. Top. Quantum Electron. 23, 9000615 (2017).

${ }^{8}$ M. Birnbaum, "Semiconductor surface damage produced by ruby lasers," J. Appl. Phys. 36, 3688-3689 (1965).
${ }^{9}$ H. M. van Driel, J. E. Sipe, and J. F. Young, "Laser induced periodic surface structure on solids: A universal Phenomenon," Phys. Rev. Lett. 49, 1955 (1982).

${ }^{10}$ R. Le Harzic, F. Stracke, and H. Zimmermann, "Formation mechanism of femtosecond laser induced high spatial frequency ripples on semiconductors at low Fluence and high repetition rate," J. Appl. Phys. 113, 183503 (2013).

${ }^{11}$ S. R. Zandbergen, R. Gibson, B. Amirsolaimani, S. Mehravar, P. Keiffer, A. Azarm, and K. Kieu, "Polarization dependent femtosecond laser modification of MBE-grown III-V nanostructures on silicon," Opt. Mater. Express 7, 2102 (2017).

${ }^{12}$ H.-J. Choi, "Vapor-liquid-solid growth of semiconductor nanowires," in Semiconductor Nanostructures for Optoelectronic Devices, edited by G.-C. Yi (Springer, 2012), Chap. 1.

${ }^{13}$ K. Kieu, S. Mehravar, R. Gowda, R. A. Norwood, and N. Peyghambarian, "Label-free multi-photon imaging using a compact femtosecond fiber laser mode-locked by carbon nanotube saturable absorber," Biomed. Opt. Express 4, 2187 (2013).

${ }^{14}$ E. D. Palik and E. D. Palik, Handbook of Optical Constants of Solids (Academic Press, 1985).

${ }^{15}$ W. M. Haynes, CRC Handbook of Chemistry and Physics, 97th ed. (CRC Press, 2016).

${ }^{16}$ A. D. Bristow, N. Rotenberg, and H. M. van Driel, "Two-photon absorption and Kerr coefficients of silicon for 850-2200 nm," Appl. Phys. Lett. 90, 191104 (2007).

${ }^{17}$ D. R. Austin, K. R. P. Kafka, Y. H. Lai, Z. Wang, K. Zhang, H. Li, C. I. Blaga, A. Y. Yi, L. F. DiMauro, and E. A. Chowdhury, "High spatial frequency laser induced periodic surface structure formation in germanium by mid-IR femtosecond pulses," J. Appl. Phys. 120, 143103 (2016).

${ }^{18}$ L. V. Keldysh, "Ionization in the field of strong electromagnetic wave," Sov. Phys. JETP 20, 1307 (1965).

${ }^{19}$ P. P. Pronko, P. A. VanRompay, C. Horvath, F. Loesel, T. Juhasz, X. Liu, and G. Mourou, "Avalanche ionization and dielectric breakdown in silicon with ultrafast laser pulses," Phys. Rev. B 58, 2387 (1998).

${ }^{20}$ H. M. Reinhardt, K. Bucker, and N. A. Hampp, "Directed assembly of gold nanowires on silicon via reorganization and simultaneous fusion of randomly distributed gold nanoparticles," Opt. Express 23, 11965 (2015).

${ }^{21}$ V. Stankevic, G. Raciukaitis, F. Bragheri, X. Wang, E. G. Gamaly, R. Osellame, and S. Juodkazis, "Laser printed nano-gratings: Orientation and period peculiarities," Sci. Rep. 7, 39989 (2017).

${ }^{22}$ A. Rudenko, J.-Ph. Colombier, S. Hohm, A. Rosenfeld, J. Kruger, J. Bonse, and T. E. Itina, "Spontaneous periodic ordering on the surface and in the bulk of dielectrics irradiated by ultrafast laser: A shared electromagnetic origin," Sci. Rep. 7, 12306 (2017).

${ }^{23}$ A. Stalmashonak, A. Podlipensky, G. Seifert, and H. Graener, "Intensitydriven, laser induced transformation of $\mathrm{Ag}$ nanospheres to anisotropic shapes,” Appl. Phys. B 94, 459 (2009).

${ }^{24}$ F. Ringleb, K. Eylers, T. Th, T. Boeck, C. Symietz, J. Bonse, S. Andree, J. Kruger, B. Heidmann, M. Schmid, and M. Lux-Steiner, "Regularly arranged indium islands on glass/molybdenum substrates upon femtosecond laser and physical vapor deposition processing," Appl. Phys. Lett. 108, 111904 (2016). 